\title{
Neonatal Diagnosis and Treatment of Menkes Disease
}

\author{
Stephen G. Kaler, M.D., M.P.H., Courtney S. Holmes, B.S., M.T., David S. Goldstein, M.D., \\ Ph.D., Jingrong Tang, M.D., Ph.D., Sarah C. Godwin, B.S., Anthony Donsante, Ph.D., \\ Clarissa J. Liew, M.D., Susumu Sato, M.D., and Nicholas Patronas, M.D. \\ Unit on Pediatric Genetics, Program in Molecular Medicine, National Institute of Child Health and \\ Human Development (S.G.K., J.T., S.C.G., A.D.), the Clinical Neurocardiology Section (C.S.H., \\ D.S.G.), and the Electroencephalography Section (C.J.L., S.S.), National Institute of Neurological \\ Disorders and Stroke, and the Imaging Sciences Program, Mark O. Hatfield Clinical Center (N.P.) \\ - all at the National Institutes of Health, Bethesda, MD. Address reprint requests to Dr. Kaler at \\ the National Institute of Child Health and Human Development, National Institutes of Health, Bldg. \\ 10, Rm. 5-2571, 10 Center Dr., MSC 1832, Bethesda, MD 20892-1832, or at kalers@mail.nih.gov
}

\section{Abstract}

BACKGROUND-Menkes disease is a fatal neurodegenerative disorder of infancy caused by diverse mutations in a copper-transport gene, $A T P 7 A$. Early treatment with copper injections may prevent death and illness, but presymptomatic detection is hindered by the inadequate sensitivity and specificity of diagnostic tests. Exploiting the deficiency of a copper enzyme, dopamine- $\beta$ hydroxylase, we prospectively evaluated the diagnostic usefulness of plasma neurochemical levels, assessed the clinical effect of early detection, and investigated the molecular bases for treatment outcomes.

METHODS-Between May 1997 and July 2005, we measured plasma dopamine, norepinephrine, dihydroxyphenylacetic acid, and dihydroxyphenylglycol in 81 infants at risk. In 12 newborns who met the eligibility criteria and began copper-replacement therapy within 22 days after birth, we tracked survival and neurodevelopment longitudinally for 1.5 to 8 years. We characterized $A T P 7 A$ mutations using yeast complementation, reverse-transcriptase-polymerase-chain-reaction analysis, and immunohistochemical analysis.

RESULTS-Of 81 infants at risk, 46 had abnormal neurochemical findings indicating low dopamine- $\beta$-hydroxylase activity. On the basis of longitudinal follow-up, patients were classified as affected or unaffected by Menkes disease, and the neurochemical profiles were shown to have high sensitivity and specificity for detecting disease. Among 12 newborns with positive screening tests who were treated early with copper, survival at a median follow-up of 4.6 years was $92 \%$, as compared with $13 \%$ at a median follow-up of 1.8 years for a historical control group of 15 latediagnosis and late-treatment patients. Two of the 12 patients had normal neurodevelopment and brain myelination; 1 of these patients had a mutation that complemented a Saccharomyces cerevisiae copper-transport mutation, indicating partial ATPase activity, and the other had a mutation that allowed some correct $A T P 7 A$ splicing.

CONCLUSIONS-Neonatal diagnosis of Menkes disease by plasma neurochemical measurements and early treatment with copper may improve clinical outcomes. Affected newborns who have mutations that do not completely abrogate ATP7A function may be especially responsive to early copper treatment.

Copyright @ 2008 Massachusetts Medical Society. All rights reserved.

No potential conflict of interest relevant to this article was reported. 
Menkes disease is an infantile-onset X-linked recessive neurodegenerative disorder caused by deficiency or dysfunction of a copper-transporting ATPase, ATP7A. ${ }^{1-3}$ The clinical and pathologic features of this condition reflect decreased activities of enzymes that require copper as a cofactor, including dopamine- $\beta$-hydroxylase, cytochrome $c$ oxidase, and lysyl oxidase. ${ }^{4}$ Recent studies indicate that ATP7A normally responds to $N$-methyl-o-aspartate receptor activation in the brain, 5,6 and an impaired response probably contributes to the neuropathology of Menkes disease. Affected infants appear healthy at birth and develop normally for 6 to 8 weeks. Subsequently, hypotonia, seizures, and failure to thrive occur, and death by 3 years of age is typical.

Treatment with daily copper injections may improve the outcome in Menkes disease if commenced within days after birth $^{7-13}$; however, newborn screening for this disorder is not available, and early detection is difficult because clinical abnormalities in affected newborns are absent or subtle. ${ }^{4,14}$ Moreover, the usual biochemical markers, low serum copper and ceruloplasmin, are unreliable predictors in the neonatal period, since levels in healthy newborns ${ }^{15}$ are low and overlap with those in infants with Menkes disease. Although molecular diagnosis is available, its use is complicated by the diversity of mutation types and the large size of $A T P 7 A(150 \mathrm{~kb}) .{ }^{16}$

A promising test for neonatal diagnosis of Menkes disease involves measurement of the neurochemicals dopamine, norepinephrine, dihydroxyphenylacetic acid, and dihydroxyphenylglycol in the plasma. ${ }^{17}$ Because of decreased activity of dopamine- $\beta$ hydroxylase, the ratio of dihydroxyphenylacetic acid to dihydroxyphenylglycol is distinctively elevated in patients with Menkes disease. ${ }^{18}$ From the known pathways of catecholamine synthesis and metabolism (Fig. 1), one would also predict a high ratio of dopamine to norepinephrine.

In addition to presymptomatic diagnosis, other challenges in the treatment of Menkes disease involve overcoming defective copper transport at two levels, the gastrointestinal tract and the blood-brain barrier, and the potential renal toxicity of exogenous copper. ${ }^{4}$ Intraperitoneal injections of copper bypass the gastrointestinal barrier and are curative in a mouse model of Menkes disease ${ }^{19}$; however, the neurodevelopmental outcomes after early subcutaneous copper injections in patients vary, ${ }^{4,7-13}$ a result implying that the human blood brain-barrier is an important hurdle. The variable outcomes after early treatment are not well understood. ${ }^{11,13,20,21}$

In this study, we prospectively evaluated the sensitivity and specificity of plasma neurochemical analysis for the diagnosis of Menkes disease, determined the clinical effect of early diagnosis and treatment, and investigated the molecular bases for treatment outcomes.

\section{METHODS} SUBJECTS

From May 1997 through July 2005, we evaluated 81 infants who were at risk for Menkes disease because of a positive family history or suggestive clinical or biochemical findings (Table 1). The study was approved by the institutional review boards of the National Institute of Child Health and Human Development and the National Institute of Neurological Disorders and Stroke. Follow-up included physical examination and neurodevelopmental assessment, or communication with referring physicians, other health care providers, or the infants' parents. Twelve of the infants who met the eligibility criteria (1 month of age or less with no neurologic symptoms) were enrolled in a clinical trial of early copper treatment reviewed by a data and safety monitoring committee. Written informed consent was obtained from parents. The study drug was copper histidine (Food and 
Drug Administration [FDA] Investigational New Drug 34,166; holder, S.G. Kaler; prepared by the National Institutes of Health [NIH] Pharmaceutical Development Service), as previously described. ${ }^{12}$ The patients visited the NIH Clinical Center approximately every 6 months for clinical and biochemical follow-up. Eight patients received treatment for 3 years; one patient, who died during the trial, received treatment for 1.6 years; and three patients under 3 years of age are still being treated. The dosage regimen was $250 \mu \mathrm{g}$ by subcutaneous injection twice daily to 1 year of age and $250 \mu \mathrm{g}$ once daily thereafter.

In a historical control group of 15 late-diagnosis patients (mean age $[ \pm \mathrm{SD}]$ at diagnosis, $163 \pm 113$ days; range, 42 to 390 ) also treated at the NIH with the same copper regimen, identification was based on classic clinical and biochemical findings (low serum copper and ceruloplasmin after the age of 3 months). ${ }^{4}$ The interval from diagnosis to initiation of copper treatment in these patients varied, depending on the time of their referral to this study. Five patients received treatment for 3 years, and 10 died before 3 years of treatment could be completed. The mean length of treatment for the latter patients was 12.2 months (range, 4.0 to 18.0$)$.

\section{ANALYSES OF PLASMA NEUROCHEMICAL VALUES AND ATP7A MUTATIONS}

Analyses of plasma neurochemical values and $A T P 7 A$ mutations were performed in our laboratory as previously described ${ }^{16,18}$ under standards and conditions certified according to the Clinical Laboratory Improvement Amendments. DNA sequencing involved $A T P 7 A$ exons and exon boundaries and was performed in both directions.

\section{YEAST COMPLEMENTATION ASSAY}

We used site-directed mutagenesis to generate the mutant $A T P 7 A$ allele $\mathrm{N} 1304 \mathrm{~S}$, as previously described. ${ }^{22}$ Other mutant constructs were prepared in the same fashion, and DNA cassettes containing sequences of interest were ligated into a yeast expression vector, MNKpYES6/CT, with fidelity confirmed by sequencing. We used wild-type and ccc2 deletion (CCC2 copper-transport deletion ${ }^{23}$ ) strains of Saccharomyces cerevisiae; the ccc2 deletion strain was from the Saccharomyces Genome Deletion Project. ${ }^{24}$ Transformation of $\operatorname{ccc} 2$ deletion and complementation experiments were performed as previously described. ${ }^{22}$

\section{IMMUNOHISTOCHEMICAL ANALYSIS AND CONFOCAL MICROSCOPY}

Dermal fibroblasts were fixed on glass slides with $4 \%$ paraformaldehyde. Blocking was performed with $3 \%$ goat serum at room temperature for 1 hour. Samples were incubated with a rabbit antibody to the carboxyl terminus of mouse $A T P 7 A$ and a Golgi marker, NBD $\mathrm{C}_{6}$-ceramide (Molecular Probes), at dilutions of 1:2000 and 1:500, respectively, at $4{ }^{\circ} \mathrm{C}$ overnight, and incubated with Texas Red-labeled antirabbit IgG antibody (Molecular Probes) at room temperature for 2 hours. Cells were viewed by a confocal microscope (Eclipse, Nikon), and images were captured by Confocal Assistant software.

\section{REVERSE-TRANSCRIPTASE-POLYMERASE-CHAIN-REACTION ANALYSIS}

Fibroblast RNA was extracted with an RNeasy Kit (Qiagen). First-strand synthesis was carried out with an Enhanced Avian First Strand Synthesis Kit (Sigma). Reversetranscriptase-polymerase-chain-reaction (RT-PCR) analysis for mRNA was carried out with primers $5^{\prime}$-GAAGGATCGGTCAGCAAGTC-3' from exon 8 (forward) and $5^{\prime}$ GGTTGCCAGCACAATCAGTA-3' from exon 10 (reverse), which generated a normal 363-bp product. 


\section{NEUROLOGIC OUTCOME MEASURES}

We obtained baseline and follow-up electroencephalograms for the 12 infants enrolled in the early-treatment trial. Most patients underwent four or more electroencephalograms. Tracings were considered abnormal if any of the following findings were present: background slowing or disorganization, spike waves or polyspikes, or diffuse spike-and-slow-wave complexes.

We obtained magnetic resonance scans of the brain using $\mathrm{T}_{1^{-}}$and $\mathrm{T}_{2}$-weighted techniques as well as flow-attenuated inversion recovery. Patients underwent up to three scans. We evaluated these studies for the presence of structural brain abnormalities and progression of white-matter myelination.

We used the Denver Developmental Screening Test II to track neurodevelopment in four areas. These were gross motor, language, fine motor-adaptive, and personal-social. ${ }^{25}$

\section{STUDY DESIGN AND STATISTICAL ANALYSIS}

All authors vouch for the accuracy and completeness of the reported data. Frontier Science and Technology Research Foundation performed the statistical analyses. Three paired comparisons were made of mean neurochemical levels and ratios: all affected versus all unaffected infants (46 and 35 infants, respectively), older (>1 month) affected versus older unaffected infants (32 and 13 infants, respectively), and newborn ( $\Delta$ month) affected versus newborn unaffected infants (14 and 22 infants, respectively). Group means were compared by nonparametric Wilcoxon rank-sum tests. A two-tailed $\mathrm{P}$ value less than 0.05 was considered to indicate statistical significance. Receiver-operating-characteristic (ROC) curves were determined for plasma dopamine, plasma dihydroxyphenylacetic acid, the ratio of dopamine to norepinephrine, and the ratio of dihydroxyphenylacetic acid to dihydroxyphenylglycol as independent diagnostic tests for Menkes disease in the 36 at-risk newborns ( $\leq$ month of age at screening). Our earlier work ${ }^{18}$ suggested that ratios of catechol levels distinguished between affected and unaffected infants as well as or better than did levels of individual catechols. Therefore, in this study we based diagnostic determinations on the ratios; a ratio of dihydroxyphenylacetic acid to dihydroxyphenylglycol of 4.0 or less was predefined as a negative test result and a value of more than 4.0 was predefined as a positive test result. The cutoff date for data acquisition in the current study was January 15, 2007.

\section{RESULTS}

\section{PROSPECTIVE DIAGNOSIS WITH PLASMA CATECHOLAMINE ANALYSIS}

The mean plasma concentrations of dihydroxyphenylacetic acid, dopamine, norepinephrine, and dihydroxyphenylglycol differed between infants at risk in whom the Menkes disease phenotype did not develop (35 infants) and those subsequently determined to have Menkes disease according to clinical, biochemical (low serum copper and ceruloplasmin at $>3$ months of age), or molecular criteria (46 infants) $(\mathrm{P}<0.001$ for all comparisons) (Table 1). In affected as compared with unaffected patients, dopamine and dihydroxyphenylacetic acid levels were higher and norepinephrine and dihydroxyphenylglycol levels were lower. The ratios of dopamine to norepinephrine and dihydroxyphenylacetic acid to dihydroxyphenylglycol differed between affected and unaffected infants $(\mathrm{P}<0.001$ for both comparisons).

Among at-risk newborns 1 month old or less (Table 1), a scatter plot of dopamine-tonorepinephrine versus dihydroxyphenylacetic acid-to-dihydroxyphenylglycol ratios (Fig. 1A) clearly distinguished affected from unaffected infants. ROC analyses showed a C 
statistic of 1.0 for plasma dopamine and 0.96 for plasma dihydroxyphenylacetic acid (Fig. 1B), results indicating high sensitivity and specificity.

\section{MOLECULAR AND BIOCHEMICAL FINDINGS IN ASYMPTOMATIC NEWBORNS}

Of 14 newborn infants with high ratios of both dopamine to norepinephrine and dihydroxyphenylacetic acid to dihydroxyphenylglycol, 12 were enrolled in a clinical trial of daily copper injections. The two nonenrolled infants were born outside the United States; the family of one of these patients declined treatment, and the other patient received copperinjection treatments at his local hospital. Subsequent biochemical and clinical findings indicated the diagnosis of Menkes disease in these patients, both of whom died within the first 2 years of life. We did not perform mutation analysis on these two infants.

Table 2 summarizes the mutations and post-treatment biochemical data for the 12 patients in the clinical trial. We identified 10 different mutations. The serum copper levels achieved were higher than those in infants with untreated Menkes disease ${ }^{26}$ in all but two patients (Patients 2 and 11). All patients tested showed increased levels of urinary $\beta_{2}$-microglobulin, a sensitive marker of renal tubular damage. ${ }^{27}$

\section{SURVIVAL IN INFANTS RECEIVING EARLY DIAGNOSIS AND TREATMENT}

We compared survival among the cohort receiving early diagnosis and treatment (mean age at first treatment, $10 \pm 4$ days) with that among a historical control group of 15 patients with Menkes disease also followed at the NIH Clinical Center who received a diagnosis and were treated later in infancy (mean age at diagnosis, $163 \pm 113$ days). Survival was $92 \%$ after a median follow-up of 4.6 years among patients treated with copper early and $13 \%$ after a median follow-up of 1.8 years among the historical control group of patients who were treated late.

\section{NEUROLOGIC OUTCOMES}

Electroencephalographic abnormalities were detected in 7 of 12 patients (58\%), although only 2 had evidence of clinical seizures. Abnormal findings included background slowing or disorganization, focal slowing (mainly in the posterior head region), focal spike waves or polyspikes (predominantly in the occipital region), and diffuse spike-and-slow-wave complexes. Distinct abnormalities on brain magnetic resonance imaging (MRI), including cortical cerebral and cerebellar atrophy and delayed myelination, were evident in six of nine patients studied. Two patients (Patients 5 and 8 ) had normal serial brain MRI scans.

We noted a range of neurodevelopmental outcomes in the early-treatment cohort (Fig. 2). Two patients had completely normal neurodevelopment during the 3-year treatment period and beyond. At their present ages ( 7 and 5 years), they have normal neurologic function, are bright and active children, and do not require special education. The mutations associated with these outcomes are IVS9,DS, $+6 \mathrm{~T} \rightarrow \mathrm{G}$ (Patient 5) and G666R (Patient 8). An unrelated patient with the G666R mutation (Patient 4) had a less successful clinical response (Fig. $2 \mathrm{~A}$ ), although his neurodevelopmental achievements clearly exceed those attained by his deceased maternal relative. At his present age ( 8 years), he rides a tricycle, walks with support, and is very socially interactive. He showed no distinctive electroencephalographic or brain MRI abnormalities (data not shown). In contrast, patients with large or small deletions that disrupt the $A T P 7 A$ translational reading frame (Patients 1, 2, 3, 6, 7, 9, 11, and 12) and one with a mutation that introduces a premature termination (or nonsense) codon (Patient 10) had less dramatic responses to early treatment, and one died (Patient 1). 


\section{CHARACTERIZATION OF MUTATIONS}

To further address the variability in response to early copper treatment among these patients, we characterized several representative mutations. These included deletion of exon 1 (Patient 2), deletion of exons 20 to 23 (Patient 12), the missense mutation G666R (Patients 4 and 8), and the IVS9 splice-junction alteration (Patient 5). We did not characterize Q1385X (Patient 10), since the predicted consequence of this nonsense mutation is similar to that of deletion of exons 20 to 23 .

RT-PCR experiments using fibroblast RNA from Patient 5 and his profoundly impaired older brother disclosed that the major effect of IVS9,DS, $+6 \mathrm{~T} \rightarrow \mathrm{G}$ is skipping of exon 9 (Fig. 3A). Exon 9 contains 226 bp of the $A T P 7 A$ sequence, which encode the first two transmembrane segments in the gene product. ${ }^{9}$ In addition to this mutant transcript, we detected a small quantity of the properly spliced transcript in both patients.

We used a yeast complementation assay ${ }^{22}$ to study the effects of deletion of exons 20 to 23 and G666R on ATP7A function. The results indicated that G666R partially complemented the $S$. cerevisiae copper-transport knockout ccc2 deletion, indicating some residual functional copper-transport capacity in this mutant allele (Fig. 3B). In contrast, the mutant allele with deletion of exons 20 to 23 failed to complement ccc2 deletion, implying absence of any residual copper-transport function. Transformation of $\operatorname{ccc} 2$ deletion with a mutant allele (N1304S) previously noted to have residual copper ATPase activity ${ }^{22}$ and with an empty vector, as positive and negative controls, respectively, gave the expected results (Fig. 3B).

Exon 1 of $A T P 7 A$ is noncoding; deletion of this exon may include the $A T P 7 A$ promoter and thus eliminate expression of the gene. ${ }^{28}$ We used immunohistochemical analysis and confocal microscopy to evaluate $A T P 7 A$ expression in cultured fibroblasts from Patient 2, who had an exon 1 deletion, and found no detectable signal (Fig. 3C).

\section{DISCUSSION}

Infants with Menkes disease who began copper treatment within the neonatal period had better survival than infants who began treatment later. To identify candidates for treatment, we exploited the copper dependence of dopamine- $\beta$-hydroxylase by measuring plasma catecholamine levels in infants at risk. Our results indicate that this approach has high sensitivity and specificity for diagnosing Menkes disease prospectively, including during its brief presymptomatic stage.

Copper is incorporated into dopamine- $\beta$-hydroxylase apoenzyme within the trans-Golgi compartment of noradrenergic neurons, a process mediated by ATP7A. In patients with congenital absence of dopamine- $\beta$-hydroxylase, the ratio of dihydroxyphenylacetic acid to dihydroxyphenylglycol is greater than 1000:1 and the ratio of dopamine to norepinephrine is approximately 50:1. ${ }^{18}$ Our results indicate that small but highly consistent elevations in both of these ratios are characteristic of Menkes disease.

We and others have reported isolated cases of Menkes disease in which early treatment with injected copper was associated with variable clinical success ${ }^{7-13}$ or with failure. ${ }^{9,28}$ On the basis of the correlations between molecular findings and clinical success presented here, we suggest that the response to early copper treatment depends on the $A T P 7 A$ genotype and that optimal outcomes occur only in patients with mutations that permit some residual copper transport. The normal neurodevelopmental outcomes in Patients 5 and 8 may reflect the combined effect of the correction of serum copper levels (Table 2) and the capacity for residual copper-transport activity associated with IVS9,DS, $+6 \mathrm{~T} \rightarrow \mathrm{G}$ and G666R, 
respectively. We speculate that this activity was sufficient for delivery of copper across the blood-brain barrier.

Patient 4, who also had the G666R mutation, showed some delay in all neurodevelopmental spheres at 36 months of age (Fig. 2), although his clinical outcome was better than that of most members of this cohort and better than that of his maternal uncle, who received a diagnosis later. Although compliance with the experimental treatment appeared to be excellent, this infant entered the trial at 22 days of age, 8 to 17 days later than all other participants. This later initiation of treatment may explain the less successful treatment outcome.

We found no evidence for residual copper transport in patients with $A T P 7 A$ deletions that disrupt the translational reading frame, and the nine infants with Menkes disease who had these mutations did not achieve normal neurodevelopment, despite early treatment.

However, these patients had better survival and a lower incidence of clinical seizures (two of nine infants, or $22 \%$ ) than would be expected among those with untreated Menkes disease, ${ }^{4}$ perhaps as a result of their access to early diagnosis and treatment. The mechanisms underlying these improvements in patients with complete loss-of-function mutations remain unclear.

More than 3 years of copper-replacement therapy for patients with Menkes disease may not be necessary or desirable. We chose a 3-year treatment period on the basis of the expected nephrotoxicity of the study drug and the knowledge that brain myelination is typically completed by 24 months of age. We found evidence of renal tubular damage associated with copper treatment in all patients tested, although this damage appears to be reversible. For example, in Patient 5, the urinary concentration of $\beta_{2}$-microglobulin reached $28.0 \mathrm{mg}$ per liter (2373.0 nmol per liter) during treatment and declined to a normal level of $0.1 \mathrm{mg}$ per liter ( $8.4 \mathrm{nmol}$ per liter) 3 years after cessation of treatment.

Patients who completed the trial have shown no declines in neurologic or cognitive skills after termination of treatment. This is also the case for a patient we previously reported on ${ }^{12}$ who remains alive and well at the age of 13 years with no neurologic or cognitive impairments 10 years after cessation of treatment.

The enhanced survival and improved outcomes associated with early diagnosis have implications regarding screening newborns for Menkes disease by measuring the blood levels of one or more of the neurochemicals measured in our study. These compounds can be detected by high-throughput tandem mass spectrometry techniques, ${ }^{29,30}$ and evaluation of their levels in dried blood spots obtained from newborns at risk could assess the suitability of this approach for mass screening.

Advances in understanding the clinical, biochemical, and molecular aspects of Menkes disease have outpaced progress in the design of therapies effective for all patients with this diagnosis. Since optimal response to copper-injection treatment appears to occur only in patients who are identified in the newborn period and whose mutations permit residual copper-transport activity, additional approaches, including $A T P 7 A$ gene transfer, are relevant future considerations.

\section{Acknowledgments}

Supported by funding from the intramural research program of the National Institutes of Health.

We thank the patients and their families for their participation, the referring clinicians for expert care and expeditious handling of plasma specimens from the infants, Alan Hinnebusch for the wild-type and ccc2 deletion strains of $S$. cerevisiae, Betty Eipper for the rabbit antibody to the carboxyl terminus of mouse $A T P 7 A$, Nina Horn 
for the fibroblast cell line from Patient II-2, Lynnette Nieman for thoughtful review of the manuscript, and Owen Rennert for many helpful discussions.

\section{REFERENCES}

1. Vulpe C, Levinson B, Whitney S, Packman S, Gitschier J. Isolation of a candidate gene for Menkes disease and evidence that it encodes a copper-transporting ATPase. Nat Genet. 1993; 3:7-13. [Erratum, Nat Genet 1993;3:273.]. [PubMed: 8490659]

2. Chelly J, Tümer Z, Tønnesen T, et al. Isolation of a candidate gene for Menkes disease that encodes a potential heavy metal binding protein. Nat Genet. 1993; 3:14-19. [PubMed: 8490646]

3. Mercer JFB, Livingston J, Hall B, et al. Isolation of a partial candidate gene for Menkes disease by positional cloning. Nat Genet. 1993; 3:20-25. [PubMed: 8490647]

4. Kaler SG. Menkes disease. Adv Pediatr. 1994; 41:263-304. [Erratum, Adv Pediatr 1995;42:xxxi.]. [PubMed: 7992686]

5. Schlief ML, West T, Craig AM, Holtzman DM, Gitlin JD. Role of the Menkes copper-transporting ATPase in NMDA receptor-mediated neuronal toxicity. Proc Natl Acad Sci U S A. 2006; 103:14919-14924. [PubMed: 17003121]

6. Schlief ML, Craig AM, Gitlin JD. NMDA receptor activation mediates copper homeostasis in hippocampal neurons. J Neurosci. 2005; 25:239-246. [PubMed: 15634787]

7. Nadal D, Baerlocher K. Menkes' disease: long-term treatment with copper and D-penicillamine. Eur J Pediatr. 1988; 147:621-625. [PubMed: 3181204]

8. Sherwood G, Sarkar B, Kortsak AS. Copper histidinate therapy in Menkes' disease: prevention of progressive neurodegeneration. J Inherit Metab Dis. 1989; 12(Suppl 2):393-396. [PubMed: 2512453]

9. Kaler SG, Buist NRM, Holmes CS, Goldstein DS, Miller RC, Gahl WA. Early copper therapy in classic Menkes disease patients with a novel splicing mutation. Ann Neurol. 1995; 38:921-928. [PubMed: 8526465]

10. Tümer Z, Horn N, Tønnesen T, Christodoulou J, Clarke JT, Sarkar B. Early copper-histidine treatment for Menkes disease. Nat Genet. 1996; 12:11-13. [PubMed: 8528242]

11. Kaler SG. Menkes disease mutations and response to early copper histidine treatment. Nat Genet. 1996; 13:21-22. [PubMed: 8673098]

12. Kaler SG, Das S, Levinson B, et al. Successful early copper therapy in Menkes disease associated with a mutant transcript containing a small in-frame deletion. Biochem Mol Med. 1996; 57:37-46. [PubMed: 8812725]

13. Ambrosini L, Mercer JF. Defective copper-induced trafficking and localization of the Menkes protein in patients with mild and copper-treated classical Menkes disease. Hum Mol Genet. 1999; 8:1547-1555. [PubMed: 10401004]

14. Gunn TR, MacFarlane S, Phillips LI. Difficulties in the neonatal diagnosis of Menkes' kinky hair syndrome — trichopoliodystrophy. Clin Pediatr (Phila). 1984; 23:514-516. [PubMed: 6467787]

15. Lockitch G, Halstead AC, Wadsworth L, Quigley G, Reston L, Jacobson B. Age- and sex-specific pediatric reference intervals and correlations for zinc, copper, selenium, iron, vitamins A and E, and related proteins. Clin Chem. 1988; 34:1625-1628. [PubMed: 3402069]

16. Liu P-C, McAndrew PE, Kaler SG. Rapid and robust screening of the Menkes disease/occipital horn syndrome gene. Genet Test. 2002; 6:255-260. [PubMed: 12537648]

17. Kaler SG, Gahl WA, Berry SA, Holmes CS, Goldstein DS. Predictive value of plasma catecholamine levels in neonatal detection of Menkes disease. J Inherit Metab Dis. 1993; 16:907908. [PubMed: 8295415]

18. Kaler SG, Goldstein DS, Holmes C, Salerno JA, Gahl WA. Plasma and cerebrospinal fluid neurochemical pattern in Menkes disease. Ann Neurol. 1993; 33:171-175. [PubMed: 8434878]

19. Hunt DM. Primary defect in copper transport underlies mottled mutants in the mouse. Nature. 1974; 249:852-854. [PubMed: 4858102]

20. Garnica AD, Frias JL, Rennert OM. Menkes kinky hair syndrome: is it a treatable disorder? Clin Genet. 1977; 11:154-161. [PubMed: 189959] 
21. Garnica A, Chan WY, Rennert O. Copper-histidine treatment of Menkes disease. J Pediatr. 1994; 125:336-338. [PubMed: 8093166]

22. Tang J, Robertson SP, Lem KE, Godwin SC, Kaler SG. Functional copper transport explains neurologic sparing in occipital horn syndrome. Genet Med. 2006; 8:711-718. [PubMed: 17108763]

23. Fu D, Beeler TJ, Dunn TM. Sequence, mapping and disruption of CCC2, a gene that crosscomplements the $\mathrm{Ca}(2+)$-sensitive phenotype of csg1 mutants and encodes a P-type ATPase belonging to the $\mathrm{Cu}(2+)$-ATPase subfamily. Yeast. 1995; 11:283-292. [PubMed: 7785328]

24. Winzeler EA, Shoemaker DD, Astromoff AH, et al. Functional characterization of the S. cerevisiae genome by gene deletion and parallel analysis. Science. 1999; 285:901-906. [PubMed: 10436161]

25. Frankenburg WK, Dodds J, Archer P, Shapiro H, Bresnick B. The Denver II: a major revision and restandardization of the Denver Developmental Screening Test. Pediatrics. 1992; 89:91-97. [PubMed: 1370185]

26. Kaler SG, Gallo LK, Proud VK, et al. Occipital horn syndrome and a mild Menkes phenotype associated with splice site mutations at the MNK locus. Nat Genet. 1994; 8:195-202. [PubMed: 7842019]

27. de Gislain C, Dumas M, d'Athis P, Lautissier J-L, Escousse A, Guerrin J. Urinary $\beta 2$ microglobulin early indicator of high dose cis-diammine-dichloroplatinum nephrotoxicity? Influence of furosemide. Cancer Chemother Pharmacol. 1986; 18:276-279. [PubMed: 3542269]

28. Liu PC, Chen YW, Centano J, Quezado M, Lem KE, Kaler SG. Downregulation of myelination, energy, and translational genes in Menkes disease brain. Mol Genet Metab. 2005; 85:291-300. [PubMed: 15923132]

29. Schulze A, Lindner M, Kohlmüller D, Olgemöller K, Mayatepek E, Hoffmann GF. Expanded newborn screening for inborn errors of metabolism by electrospray ionization-tandem mass spectrometry: results, outcome, and implications. Pediatrics. 2003; 111:1399-1406. [PubMed: 12777559]

30. Hao C, March RE, Croley TR, Chen S, Legault MG, Yang P. Study of the neurotransmitter dopamine and the neurotoxin 6-hydroxydopamine by electrospray ionization coupled with tandem mass spectrometry. Rapid Commun Mass Spectrom. 2002; 16:591-599. [PubMed: 11870897] 


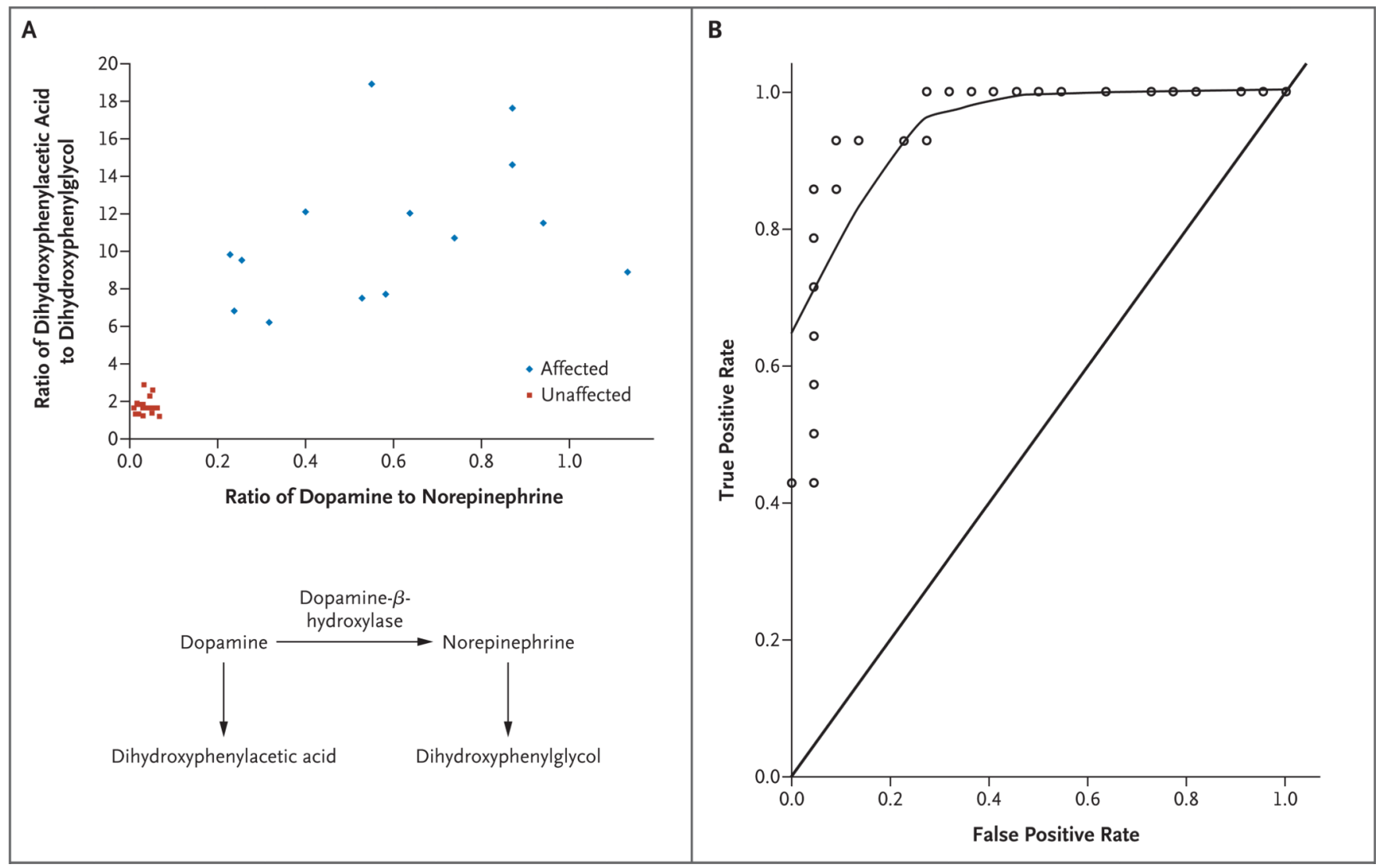

Figure 1. Diagnostic Value of Plasma Neurochemicals for Neonatal Detection of Menkes Disease Panel A is a scatter plot of the ratio of plasma dopamine to norepinephrine versus the ratio of plasma dihydroxyphenylacetic acid to dihydroxyphenylglycol in 36 newborns at risk. Partial deficiency of dopamine- $\beta$-hydroxylase in Menkes disease ${ }^{18}$ predicts a buildup of proximal metabolites in the normal catecholamine biosynthetic pathway and decreased levels of the distal metabolites. The ratios of dopamine to norepinephrine and of dihydroxyphenylacetic acid to dihydroxyphenylglycol reflect these alterations and distinguish the 14 affected infants from the 22 unaffected infants. The normal pathway of catecholamine biosynthesis is shown below the graph. Panel B shows a receiver-operatingcharacteristic (ROC) curve for plasma dihydroxyphenylacetic acid in 36 newborns at risk for Menkes disease. ROC curves show the relationship between true positive and false positive rates for a test across various threshold values used to diagnose a condition. The upper curve, plotted by the locally weighted scatter-plot smoothing technique, represents the sensitivity and specificity for the diagnosis of Menkes disease when different cutoff values for dihydroxyphenylacetic acid are applied. The area under the curve ( $\mathrm{C}$ statistic) for the ROC shown is 0.96 . The diagonal line indicates where the curve would rest if a test were completely unreliable (area under the curve, 0.5). The $\mathrm{C}$ statistic for plasma dopamine levels in this sample was 1.0 (data not shown). 


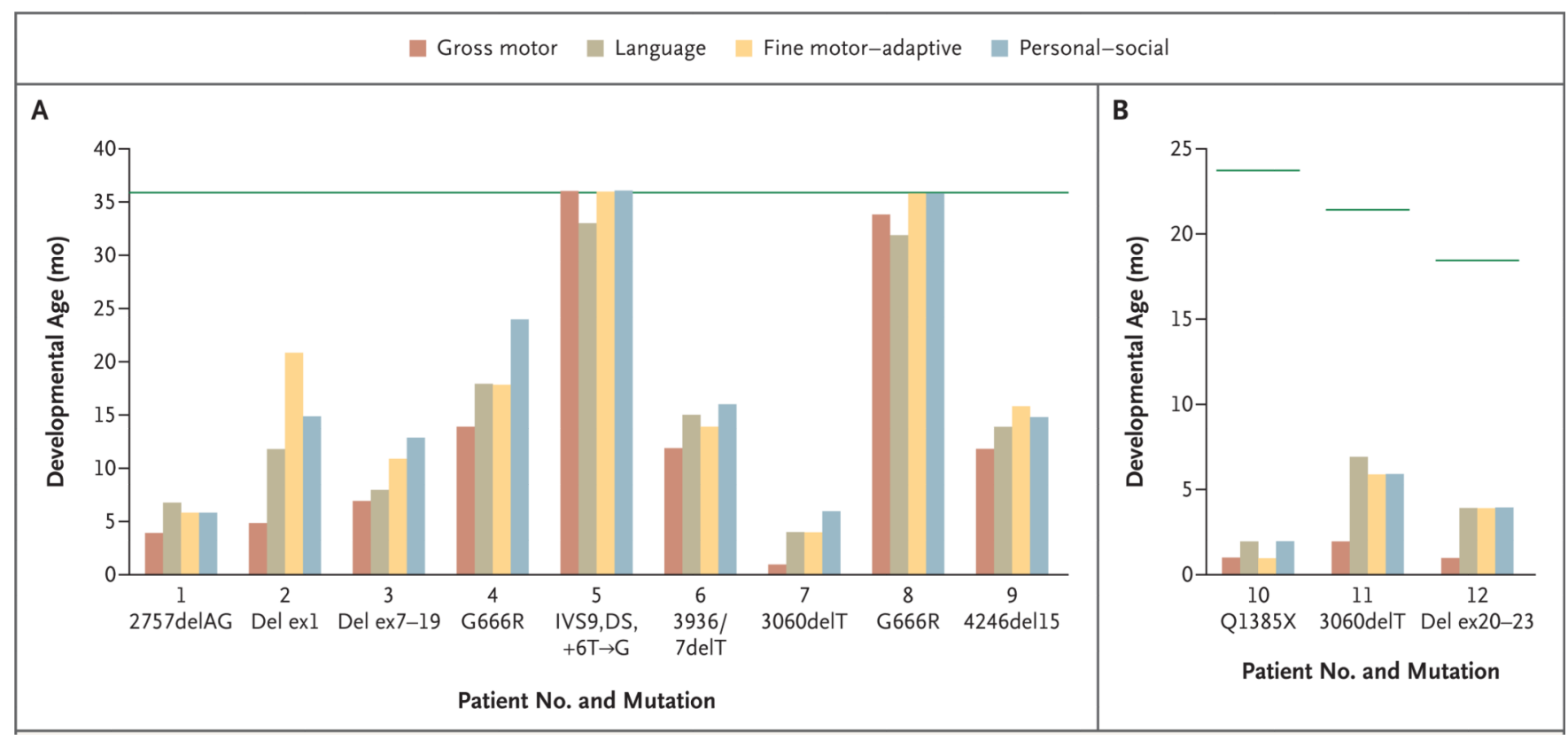

Figure 2. Neurodevelopmental Outcomes in Patients Who Received a Diagnosis of Menkes Disease as Newborns

Clinical neurodevelopmental outcomes at 36 months of age are shown for Patients 1 to 9 (Panel A). Patient 1 died at 19 months of age. The current neurodevelopmental levels are shown for Patients 10, 11, and 12 at less than 36 months of age (Panel B). The horizontal green lines show the normal level of development for the patient's age. Del denotes deletion, and ex exon. 


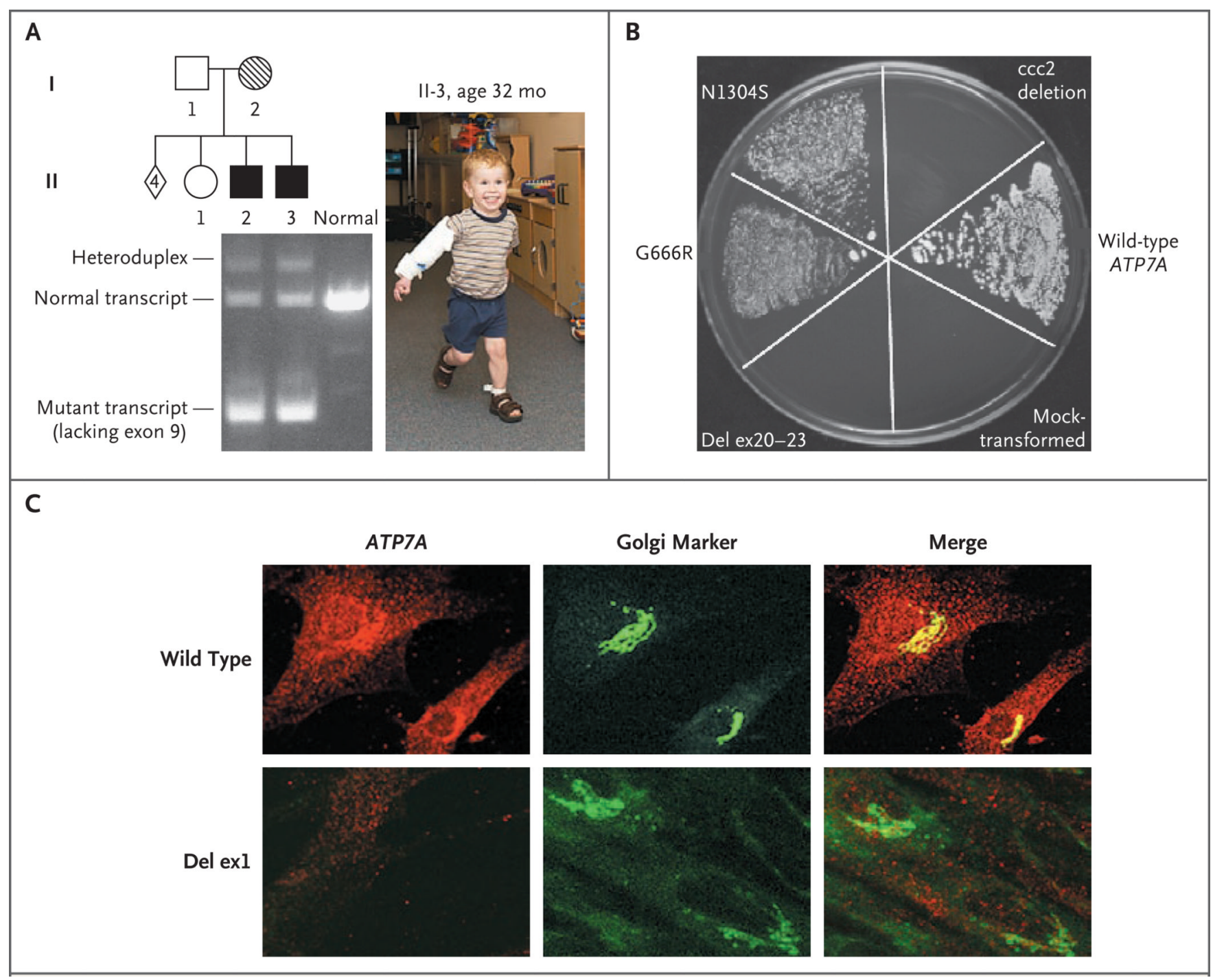

Figure 3. Characterization of ATP7A Mutations in an Early-Treatment Cohort of Patients with Menkes Disease

Panel A shows reverse-transcriptase-polymerase-chain-reaction analysis of fibroblast mRNA in a treatment-responsive patient with an IVS9,DS, $+6 \mathrm{~T} \rightarrow \mathrm{G}$ mutation. The patient (II-3 in the pedigree), shown at the age of 32 months, and his older, profoundly disabled brother (II-2), who did not receive an early diagnosis or treatment, both manifested a large quantity of a mutant transcript lacking exon 9, a smaller quantity of the properly spliced 363-bp transcript, and a heteroduplex of both species showing delayed electrophoretic migration. In the pedigree, squares indicate males, circles indicate females, and the diamond indicates four miscarriages. Solid symbols denote affected family members, open symbols unaffected family members, and hatch marks a known carrier of the mutation. In Panel $\mathrm{B}$, a yeast complementation assay distinguishes $A T P 7 A$ alleles. The plating pattern (clockwise from the 12 o'clock position) includes the yeast copper-transport mutant ccc 2 deletion; $\operatorname{ccc} 2$ deletion transformed with the normal (wild-type) $A T P 7 A$ allele; ccc2 deletion mocktransformed with an empty vector; ccc 2 deletion transformed with a mutant $A T P 7 A$ allele harboring deletion of exons 20 to 23; ccc2 deletion transformed with a mutant $A T P 7 A$ allele, G666R; and ccc2 deletion transformed with the mutant $A T P 7 A$ allele, N1304S (associated with typical occipital-horn syndrome, a mild variant of Menkes disease, and 
used as a positive control22). Yeast strains were plated on four different mediums: normal yeast extract-peptone-dextrose (YPD), synthetic yeast nitrogen base (YNB) supplemented with copper ("copper-sufficient" medium), YNB supplemented with iron ("iron-sufficient" medium), and copper- and iron-limited YNB. All strains grew on YPD, copper-sufficient, and iron-sufficient mediums (data not shown), whereas only the wild type, G666R, and N1304S grew on the copper- and iron-limited medium, indicating copper-transport activity associated with these alleles. The wild-type allele shows the most robust growth. The allele with the deletion of exons 20 to 23 failed to complement the knockout strain, indicating no residual copper-transport activity. Panel $\mathrm{C}$ shows confocal microscopical images of fibroblasts from a normal male infant and from Patient 2 (deletion of exon 1) stained with a C-terminal antibody to murine ATP7A (red) and the Golgi marker NBD $\mathrm{C}_{6}$-ceramide (green). In normal control fibroblasts (wild type), there is direct overlap between the ATP7A and Golgi signals (Merge panel), whereas in the cells with the deletion of exon 1, there is no visible ATP7A signal. Del denotes deletion, and ex exon. 


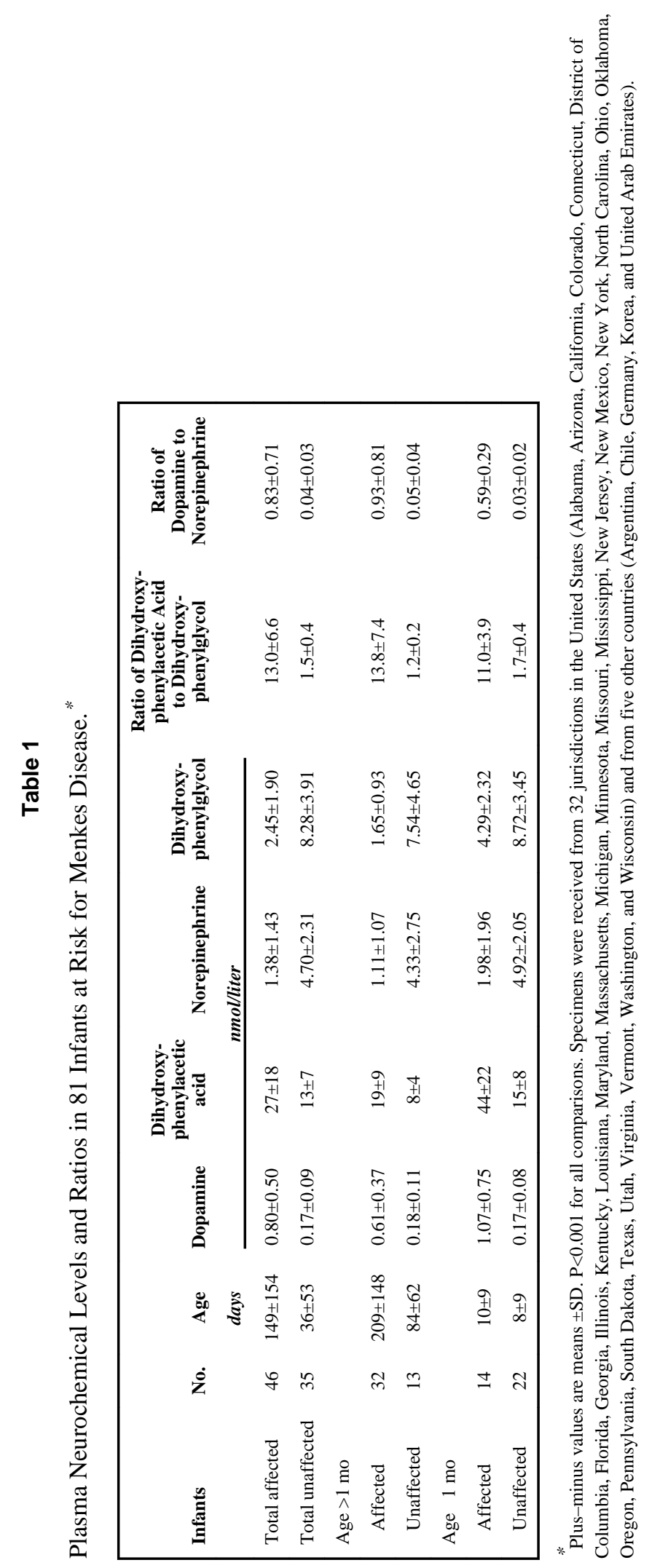

NEngl J Med. Author manuscript; available in PMC 2012 October 21. 
